\title{
Learned voluntary control of heart rate and rhythm in two subjects with premature ventricular contractions ${ }^{1}$
}

\author{
THOMAS G. PICKERING² AND NEAL E. MILLER \\ From the Rockefeller University, New York, N.Y. 10021, U.S.A.
}

\begin{abstract}
Two people with frequent premature ventricular contractions and bigeminal rhythm not associated with other signs of heart disease, learned to suppress the arrhythmia using a biofeedback technique. In both cases, physical exercise and atropine suppressed the arrhythmia, and in both the learned control was related to the development of tachycardia. In Case 1, it appeared to depend on something more than speeding alone, for sinus rhythm could be maintained during voluntary control at a lower heart rate than during physical manoeuvres such as exercise. In Case 2, the premature ventricular contractions could be induced by a sudden fall of heart rate, e.g. after a Valsalva manoeuvre, and could be suppressed by voluntary increase of heart rate by up to 40 beats/minute produced with little change in breathing or muscle activity.
\end{abstract}

The treatment of premature ventricular contractions in a patient with an otherwise normal heart is often very unsatisfactory. If the premature ventricular contractions are troublesome enough to warrant treatment, the decision to use antiarrhythmic drugs is influenced by the fact that not only are the drugs often ineffective, but also dangerous. Nearly all the drugs in common use for this purpose act directly on the heart, but there is increasing evidence, reviewed by Mauck and Hockman (1967), that the central nervous system may influence the appearance and disappearance of premature ventricular contractions. Recent work (Lown et al., 1973a; Pickering et al., 1976) has shown that the frequency of these contractions often falls during sleep, and, conversely, that ventricular tachyarrhythmias may be precipitated by psychological stress (Lown et al., 1973b).

One possible alternative mode of treatment has been proposed by Weiss and Engel (1971), who found that some patients with frequent premature ventricular contractions were able to learn to control them voluntarily. The training consisted of learning to produce small changes of heart rate in both directions, using a biofeedback technique where a yellow light signalled the correct response. In 5 out of 8 patients, the acquisition of this ability was found

\footnotetext{
${ }^{1}$ Supported by grants from the National Institute of Mental Health and from the Wellcome Trust.

'Present address: Cardiovascular Center, New York Hospital, Cornell University Medical Center, New York, N.Y. 10021, USA. Received for publication 17 June 1976
}

to be associated with a decreased frequency of premature ventricular contractions, which appeared to be maintained outside the laboratory situation. The present work, a preliminary report of which has already been published (Pickering and Miller, 1975), was undertaken to examine further the possibility of direct voluntary control of premature ventricular contractions, and to investigate the mechanisms of such control. Two patients were studied, who will be described separately because there were considerable differences both in the manifestations of the contractions and in the type of training used.

\section{Case 1}

\section{PREVIOUS HISTORY}

A 14-year-old boy who had always been in good health and free of any cardiac symptoms, was noted to have an irregular pulse $2 \frac{1}{2}$ years previously on a routine medical examination, but no other abnormality was found. The electrocardiogram showed frequent premature ventricular contractions: because these often occurred in runs of three or more, it had been decided that they should be treated. At rest, however, the rhythm was usually bigeminal, interspersed with brief runs of sinus rhythm. For various reasons, treatments with lignocaine, quinidine, or propranolol were all unsuccessful. Pharmacological tests were carried out at the University of Pennsylvania Medical School by Dr. T. Weiss. These showed that intravenous phenylephrine, phentolamine, propranolol, edrophonium, lignocaine, and isoprenaline all failed to suppress the 
premature ventricular contractions. Particularly noteworthy is the fact that with isoprenaline they persisted unchanged up to a heart rate of 143/ minute. Only atropine abolished them, above a rate of 110 /minute.

He had no symptoms directly attributable to his arrhythmia and was unable to tell whether he was in sinus or bigeminal rhythm.

\section{METHODS AND PROCEDURE}

The principal aim of the training procedure was to enable the patient to develop voluntary control over his heart rate or rhythm. Since he was not normally aware of either, an appropriate change of the variable to be controlled was signalled to him as soon as it occurred. Learning a physical skill (such as heart rate control, or riding a bicycle) is often largely a matter of trial and error. While knowledge of results (success or failure) is essential for learning to take place, it is not necessary to know exactly how or why the correct response is produced. One cannot learn to ride a bicycle simply by reading a book about it. Thus, no particular instructions could be given as to how the patient should succeed, but verbal encouragement was given whenever there was a change in the required direction. To begin with, such changes are largely spontaneous, but later one can be produced voluntarily. Heart rate training was initially tried using a method similar to the one described by Weiss and Engel (1971), but because the subject was in bigeminal rhythm most of the time while at rest, this proved unsatisfactory, and it was found easier to give the feedback in the form of the electrocardiographic trace displayed on the oscilloscope screen of a cardiac monitor. With the electrode configuration used, the difference between the sinus beats and the premature ventricular contractions was obvious.

Training was continued over a period of 6 months. A total of 115 'trials' (defined below) was performed, and there were usually 4 trials per session. At the beginning of each session, four standard procedures were carried out. These were: (a) Valsalva's manoeuvre, straining at $40 \mathrm{mmHg}$ for 10 seconds, (b) hyperventilation for 45 seconds, (c) breath-holding for $\mathbf{4 5}$ seconds, and (d) isometric hand grip for $\mathbf{4 5}$ seconds.

Respiration was recorded by a pneumograph round the chest connected to a low-pressure transducer. Palmar galvanic skin conductance was also recorded. Records were taken on an 8-channel Grass polygraph. The training procedure was done with the subject lying supine on a bed, facing the oscilloscope screen. The recording apparatus was in an adjoining room out of sight of the subject. The control periods (4 minutes) were done with the oscilloscope screen disconnected, and the subject lying at rest. During the training periods, or trials (usually 13 minutes), he watched the screen and was instructed to maintain a pattern of normal rhythm for as much time as he could. He was told that it would be better if he did this without changing his breathing or tensing his muscles, but no particular emphasis was placed on this. Some trials without feedback were done with the oscilloscope disconnected. His ability to detect changes of rhythm was tested at intervals by asking him to press a button (which produced a mark on the paper record) every time he thought his rhythm had changed from sinus to bigeminal and vice versa. He was never able to do this consistently.

The results were analysed by classifying the rhythm as sinus, bigeminy, or trigeminy, and expressed as the percentage of time in each rhythm during each control or trial period.

\section{Results}

\section{EFFECTS OF PHYSICAL MANOEUVRES} ON RHYTHM

While resting on the bed between the four standard procedures, he was normally in bigeminal rhythm. Hyperventilation, isometric hand grip, and Valsalva manoeuvres tended to produce runs of sinus rhythm, following an increase in the sinus rate. Dynamic bicycle exercise was also carried out on two occasions. On the first (three weeks after the start of training), the premature ventricular contractions were suppressed above a sinus rate of $156 /$ minute. On the second (after 10 weeks of training), the contractions disappeared above a rate of 129 /minute.

\section{LEARNED CHANGES OF RHYTHM \\ 13-minute trials}

An ability to increase the amount of sinus rhythm was not at first apparent and was only acquired after trial 9. Before this trial, there was no significant difference between the amount of sinus rhythm during the control periods (mean $2.9 \%$ ) and the trial periods (mean $5 \cdot 2 \%$ ), but after trial 10, a consistent difference between the two was apparent. Thus, for the next 9 trials, sinus rhythm was present for 6.2 per cent of the control time and 32.7 per cent of the trial time, and this difference was significant $(t=5.01, P<0.001)$. Comparison of the first 10 trials of training with the last 10 (done 6 months later), showed that during the first 10 sinus rhythm was present 5.3 per cent of the time during rest, and 8.7 per cent during training, a difference of only 3.4 per cent. For the last 10 trials, the percentage of time in sinus rhythm was 
3.2 per cent at rest and 27.4 per cent during the trial periods, a difference of $24 \cdot 2$ per cent. He thus showed the development of voluntary control of his rhythm over a period of several months. He was not able to maintain a significant increase of sinus rhythm during 'no-feedback trials' (when the oscilloscope was disconnected).

\section{2-minute trials}

During these trials, the subject was asked to switch alternately from one rhythm to the other every two minutes. An example of such a trial is shown in Fig. 1, which is a continual strip of electrocardiogram in 2-minute segments. The subject attempted to change his rhythm at the beginning of each segment. While he is at rest ( $R$ in the Fig.), there is bigeminal rhythm. During the periods marked $S$

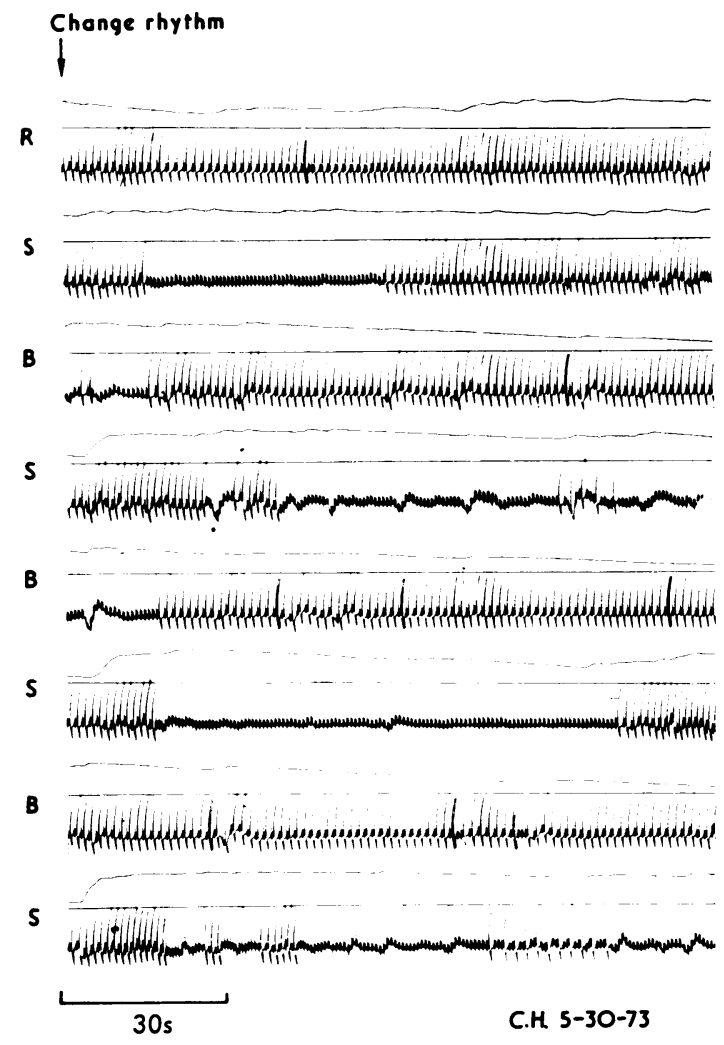

Fig. 1 Case 1. Continuous 16-minute record (in 2-minute segments) of electrocardiogram and skin conductance (upper line of each trace). $R=$ Rest. Change rhythm =instruction to change rhythm either to sinus (S) or bigeminy (B) for two minutes. Premature ventricular contractions have a large amplitude on the electrocardiogram; sinus beats are small. Note increase of conductance following $S$, but not $B$. (attempting to maintain sinus rhythm), there are periods of sinus rhythm which revert to bigeminy during the alternate periods $(B$, when he was attempting to maintain bigeminal rhythm). One noteworthy feature is that the galvanic skin conductance showed consistent changes between the two types of rhythm. During the periods of bigeminal rhythm, the skin conductance was low, whereas the beginning of each period of sinus rhythm was accompanied by an increased skin conductance, indicating sympathetic arousal. The pattern of breathing tended to be different also; during the periods of sinus rhythm it became faster and more irregular.

\section{CHANGES OF RATE DURING CHANGES}

OF RHYTHM

Since the transition from bigeminal rhythm to sinus rhythm during the physical procedures was associated with a relative tachycardia, it was of interest to know whether the same was true of the learned rhythm changes. Analysis of the voluntarily produced transition from bigeminal to sinus rhythm showed that there was a consistent speeding from an average sinus rate of $90.6 /$ minute at the end of bigeminal rhythm to $95 \cdot 6 /$ minute at the onset of sinus rhythm.

Once sinus rhythm had been achieved, however, this speeding did not appear to be maintained. The relation between heart rate and rhythm, both for the 'physical manoeuvres' described above, and for the training periods was analysed by the following method. The average sinus rates for all periods of sinus or bigeminal rhythm lasting more than 10 seconds were measured. For the physical manoeuvres, measurements were made both while the subject was at rest on the bed and while performing the various manoeuvres. Periods of intermediate rhythm, e.g. trigeminal, were excluded from this analysis. The results were plotted as histograms showing the relative prevalence of the two rhythms (sinus and bigeminal) at different heart rates. The upper part of Fig. 2 shows that during the various physical manoeuvres, sinus rhythm prevailed at higher rates, and bigeminal rhythm at lower rates.

When the data for the training periods were analysed in the same way (lower half of Fig. 2), the results were very different. The distribution of bigeminal rhythm in relation to heart rate was much the same as for the physical manoeuvres, but most of the sinus rhythm now occurred at lower heart rates than those during bigeminal rhythm. This means that the learned, voluntarily produced sinus rhythm was not simply the result of maintained speeding of the heart, as was the case with the physical manoeuvres. During the voluntarily 


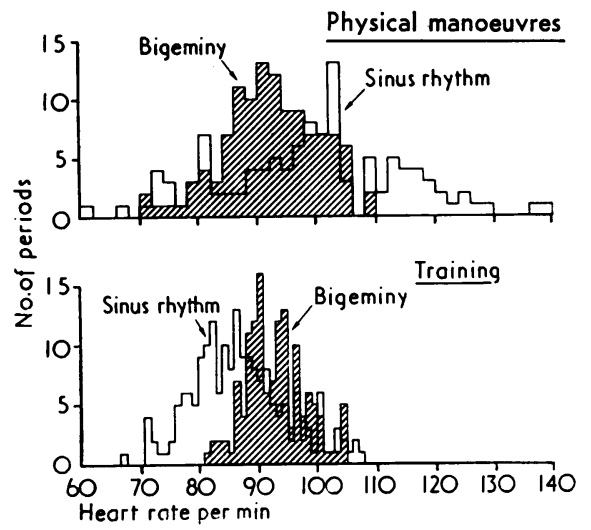

Fig. 2 Case 1. Relative prevalence of bigeminal (hatched) and sinus rhythms at different heart rates. Each period=one episode of at least 10 seconds' duration. Top panel: during physical manoeuvres; Lower panel: during trials. See text for descriptions.

produced sinus rhythm, the initial speeding associated with the change from bigeminy to sinus rhythm was followed by a period of slowing without the reappearance of bigeminal rhythm.

\section{Case 2}

\section{PREVIOUS HISTORY}

The second patient was a 35-year-old man who for one year before the study had experienced episodes of palpitation lasting for several hours at a time, which were uncomfortable and brought on feelings of fatigue and helplessness. At first, he would lie still until they eventually passed off, but found that physical exercise would sometimes suppress them.

Physical examination and the electrocardiogram showed no abnormality other than premature ventricular contractions. Most of the time these were sporadic, but during symptomatic attacks, the rhythm was bigeminal.

\section{METHODS AND PROCEDURE}

The recording procedure was the same as for Case 1, except that feedback was given in the form of a meter connected to the output channel of the Grass cardiotachograph, which was directly calibrated in beats per minute. Since the arrhythmia was more intermittent than in the first case, emphasis was placed on being able to produce short bursts of speeding, and the trials were normally for 30 seconds each.

The ability to detect premature ventricular contractions and the effects of physical manoeuvres on the arrhythmia were tested as in Case 1.
He was seen on two separate occasions 5 months apart. On each occasion, he attended for 4 days at a time and performed 4 training sessions a day, of about an hour each.

\section{Results}

\section{EFFECTS OF PHYSICAL MANOEUVRES} ON RHYTHM

While at rest on the bed, he was in sinus rhythm most of the time with sporadic premature ventricular contractions. His ability to detect these was tested as for the first subject; 10 per cent of 32 sporadic premature ventricular contractions were correctly detected, but he gave 12 false positive signals. The onset of bigeminal rhythm was always correctly identified.

Valsalva manoeuvres nearly always induced the bigeminal rhythm during the overshoot phase of the manoeuvre when there was a period of intense reflex bradycardia. The other three standard procedures did not induce the arrhythmia. Exercise on the bicycle ergometer reduced the frequency of premature ventricular contractions, which were absent above a rate of $115 /$ minute.

\section{LEARNED CHANGES OF RATE AND RHYTHM: FIRST VISIT}

When first tested, he could produce no consistent tachycardia, but by the end of the first visit ( 16 sessions), he could increase his rate by 20 to 25 / minute. During the speeding trials, there was at first a noticeable change in the respiratory pattern and in the activity of the EMG of the chin muscles. As with the first subject, palmar skin resistance decreased. The tachycardia was usually accompanied by a rise of diastolic pressure. The speeding was usually maintained for only 30 seconds, but he was able to maintain a heart rate increase of $10 /$ minute or more for 8 minutes.

\section{SUPPRESSION OF PREMATURE}

VENTRICULAR CONTRACTIONS

Once the ability to speed the heart voluntarily had been acquired, he was able to learn to suppress the bigeminal rhythm, which was first brought on by performing a Valsalva manoeuvre. A typical episode is shown in Fig. 3, which is a polygraph record of muscle tension (EMG) from the chin, heart rate, electrocardiogram, and respiration during two attempts to suppress the arrhythmia. It will be noticed that the first attempt shown here was unsuccessful, but the second suppressed the arrhythmia. The average sinus rate at the transition point to sinus rhythm was $117 \pm 8 /$ minute. One 
frequent finding at this stage can be seen in Fig. 3; he often needed two attempts to suppress the premature ventricular contractions but, as shown in the figure, the rate at which the transition finally occurred during the second attempt was usually lower ( $116 \pm 8 /$ minute) than the maximum rate achieved during the first $(123 \pm 7 /$ minute); this difference was significant $(t=4.39, P<0.002)$.

During 20 attempted suppressions of the arrhythmia, bigeminal rhythm was present for a total of 2304 seconds while he was at rest, and it stopped spontaneously only once (a few seconds after an attempted suppression). He was trying to suppress it for a total of 639 seconds, during which time it stopped 19 times. This difference was significant $\left(x^{2}=64 \cdot 0, P<0 \cdot 005\right)$. He was able to do this with or without feedback, though he was unable to tell exactly when sinus rhythm was resumed unless feedback was present.

\section{SUBSEQUENT PROGRESS}

During the following two months, he was able to suppress the bigeminal rhythm on about 50 per cent of occasions. He bought himself a cardiac monitor and practised heart-rate speeding at home. He noticed that it was harder to suppress the arrhythmia when it came on spontaneously than when he induced it himself.

\section{SECOND VISIT}

He could now increase his heart rate by 20 to 40 / minute equally well with or without feedback. With the feedback on, he was able to hold his rate very accurately at any given level between 70 and 110/minute (Fig. 4). This figure shows that EMG and respiratory changes were more pronounced at the higher heart rates, and also that sporadic premature ventricular contractions were more likely to occur during a period of rapid slowing (as seen earlier during the Valsalva manoeuvre) than at any steady level of heart rate.

He was now able to maintain sizeable increases of rate (about 20/minute) without any noticeable change in his breathing or chin EMG activity (Fig. 5). In the example shown in this figure, he increased his rate from 76 to $95 /$ minute. He could also speed his heart while holding his breath. Although the skeletal changes (i.e. breathing and EMG) could be eliminated, the associated autonomic changes, i.e. rise of skin conductance and diastolic pressure (in this instance by $10 \mathrm{mmHg}$ ) persisted.

Valsalva manoeuvres were again used to induce the bigeminal rhythm. This time he was even more successful at suppressing the arrhythmia. In 10 trials the arrhythmia did not stop at all during 1603 seconds while he was resting, but stopped $10 / 10$ times during 112 seconds while he was trying to

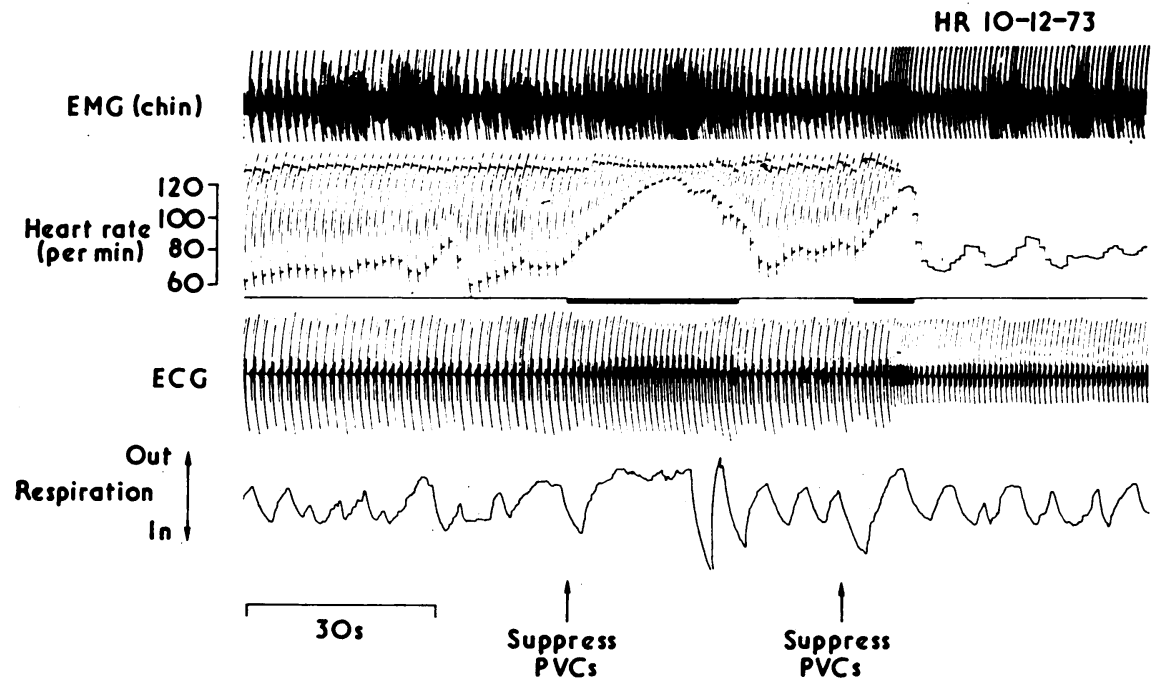

Fig. 3 Case 2. Voluntary suppression of premature ventricular contractions. Upper trace =chin EMG (with electrocardiograph artefact). Second trace = heart rate: early part of record is bigeminal rhythm, and irregularity is caused by premature beats followed by compensatory pause. Solid bars =subject presses buzzer to indicate attempted suppression, which succeeds on second attempt. 


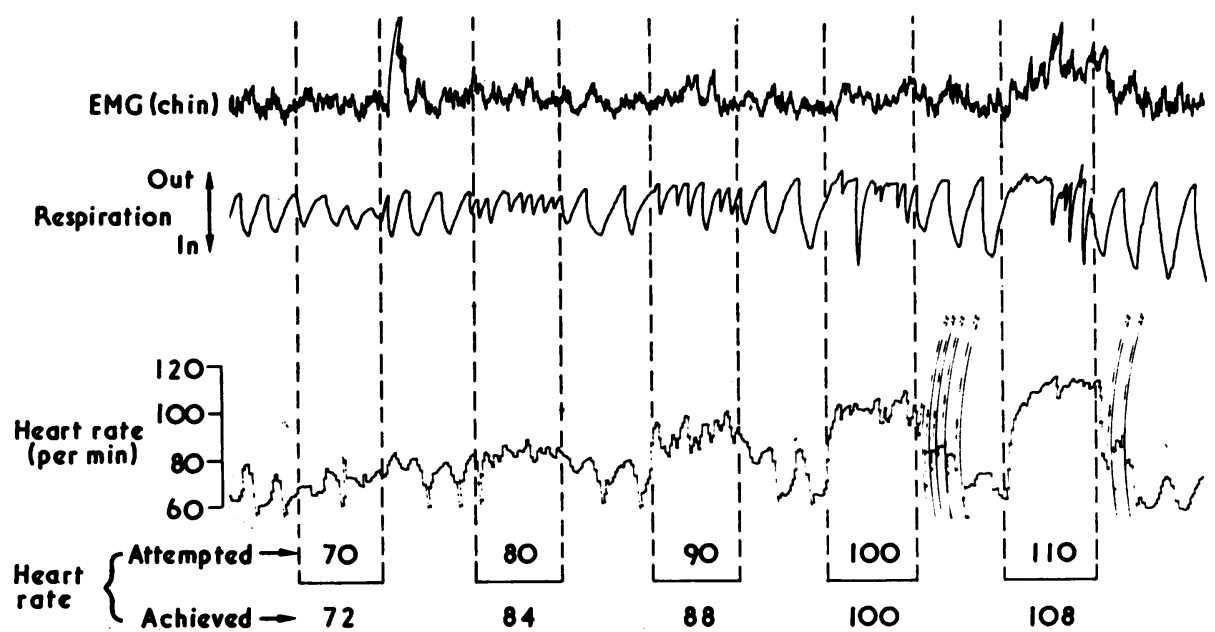

Fig. 4 Case 2. 30-second alternations of rest and speeding to different rates. Achieved=actual rate over last 15 seconds. Note increasing EMG and respiratory changes at higher rates and onset of premature ventricular contractions following the last two speeding episodes (large deflections on heart rate record).

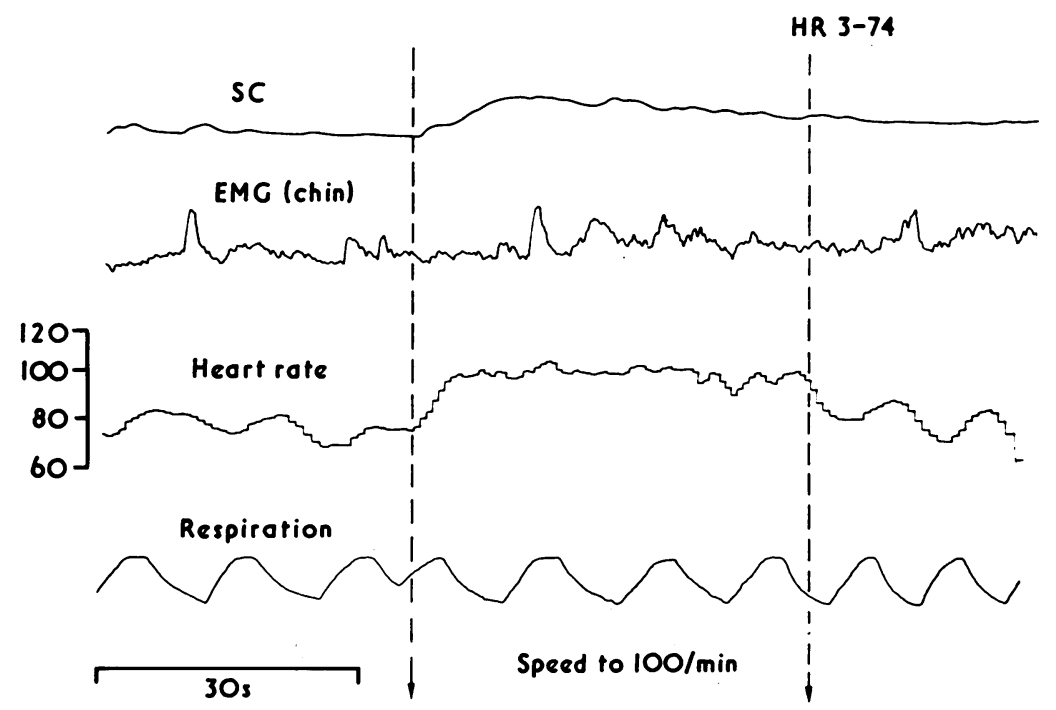

Fig. 5 Case 2. Ability to speed heart in absence of respiratory or EMG change, but change of skin conductance (SC) persists. 
suppress it. This difference was significant $\left(x^{2}=\right.$ 132.9, $P<0.005)$. He was successful on the first attempt on 9 out of the 10 trials.

\section{EFFECT OF ATROPINE}

Fig. 6 shows the effect of $2.8 \mathrm{mg}$ atropine on the voluntary tachycardia. Before it was given, he was able to produce brief 5-second bursts of acceleration having almost a square wave appearance on the heart rate trace. The rapidity of these changes suggests that they were most likely mediated vagally. After administration of atropine, the heart rate rose to 120 /minute. Despite this, he was still able to produce small and brief periods of acceleration (of 15 /minute), but their appearance was now quite different (right hand panel of Fig. 6). The peak acceleration was now not reached until after he had stopped trying to produce it, and took many seconds to decay. This pattern is consistent with a sympathetically mediated change. Valsalva manoeuvres no longer produced premature ventricular contractions, and none was seen in the hour following the atropine.

\section{Discussion}

The chief finding of these investigations is that learned voluntary control of premature ventricular contractions can be achieved, confirming the findings of Weiss and Engel (1971). The two cases described here were similar in that both occurred in young people with otherwise normal hearts, and in both the appearance and disappearance of the arrhythmia were strongly influenced by changes of heart rate. Thus in both cases, the voluntary production of sinus rhythm was preceded by an increase in the sinus rate during bigeminy. In the first case, the sinus rhythm appeared to be maintained while there was a gradual slowing of the sinus rate, for the eventual sinus rate was usually lower than the sinus rate for bigeminal rhythm. This was in obvious contrast to the speeding induced by purely physical manoeuvres, where the sinus rate was almost always higher during sinus rhythm than during bigeminy. In the second case, the transition from bigeminal to sinus rhythm was accomplished solely by speeding the heart.

In both cases, physical exercise and vagal blockade by atropine suppressed the premature ventricular contractions, though it is not clear whether this effect was primarily the result of heart rate per se rather than of vagal withdrawal. In Case 2, the voluntary speeding was probably achieved by a combination of vagal and sympathetic changes, which is consistent with earlier reported cases of voluntary tachycardia (Favill and White, 1917; West and Savage, 1918; Ogden and Shock, 1939; Pickering and Gorham 1975). This speeding did not appear to occur merely as a result of changes in muscle tension or respiration, suggesting that some degree of direct voluntary autonomic control was achieved.

To be therapeutically effective, the learned control of any disordered function must be carried over to situations outside the laboratory. The first requisite for this to occur is that the learned behaviour can be performed in the absence of external feedback. Our second case could do this, but the first was unable to do it consistently. The type of control needed for therapeutic benefit may be of two kinds, which are exemplified by our two cases. On the one hand, the disturbance may be relatively continuous and asymptomatic, as was the arrhythmia in Case 1, and as are some other disorders where biofeedback techniques are being applied (e.g. hypertension). In such disorders, the learned control must become relatively continuous, and therefore subconscious. On the other hand, the disturbance may be intermittent and symptomatic, as was the arrhythmia in Case 2 . In this type, the
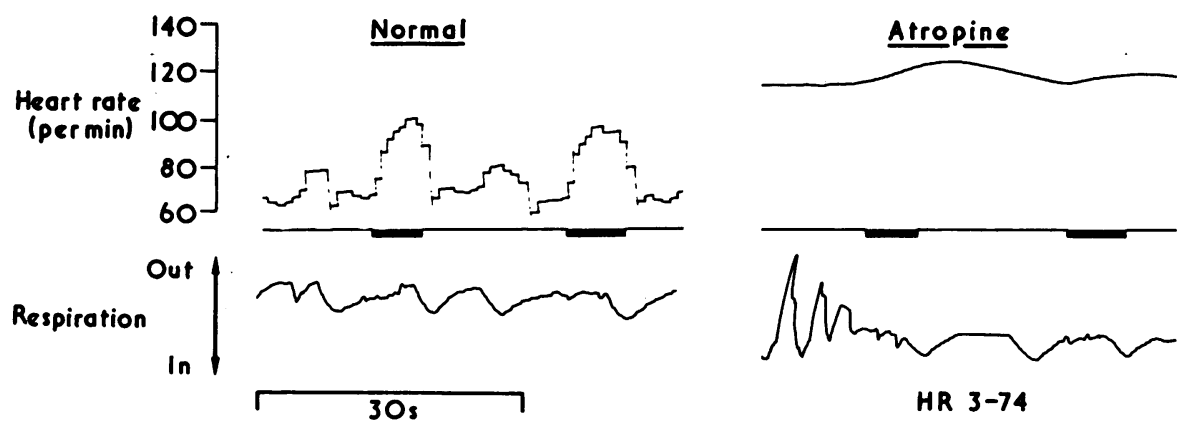

Fig. 6 Case 2. Five-second attempts at voluntary speeding (signalled by solid bars), before (left) and after (right) atropine. 
learned behaviour need only be performed when the symptoms arise, and a successful suppression of symptoms will tend to reinforce the behaviour pattern. It would be expected that this second type would respond better to biofeedback training than the first, and our two cases would tend to bear this out. It remains to be seen whether such training confers any sustained therapeutic benefit.

\section{References}

Favill, J., and White, P. D. (1917). Voluntary acceleration of the rate of the heart. Heart, 6, 175.

Lown, B., Tykocinski, M., Garfein, A., and Brooks, P. (1973a). Sleep and ventricular premature beats. Circulation. 48, 691 .

Lown, B., Verrier, R., and Corbalan, R. (1973b). Psychologic stress and threshold for repetitive ventricular response. Science, 182, 834.

Mauck, H. P., and Hockman, C. H. (1967). Central nervous mechanisms mediating cardiac rate and rhythm. American Heart fournal, 74, 96.
Ogden, E., and Shock, N. W. (1939). Voluntary hypercirculation. American fournal of the Medical Sciences, 198, 329.

Pickering, T., and Gorham, G. (1975). Learned heart-rate control by a patient with a ventricular parasystolic rhythm. Lancet, 1, 252.

Pickering, T. G., Goulding, L., Cobern, B. A., Johnston, J., and Honour, A. J. (1976). Diurnal variations of ventricular extrasystoles. Quarterly fournal of Medicine. In the press.

Pickering, T. G., and Miller, N. E. (1975). Learned voluntary control of heart rate and rhythm in two subjects with premature ventricular contractions. Clinical Science and Molecular Medicine, 49, 17P.

Weiss, T., and Engel, B. T. (1971). Operant conditioning of heart rate in patients with premature ventricular contractions. Psychosomatic Medicine, 33, 301.

West, H. F., and Savage, W. E. (1918). Voluntary acceleration of the heart beat. Archives of Internal Medicine, 22, 290.

Requests for reprints to Dr. T. G. Pickering, Cardiovascular Center, New York Hospital, Cornell University Medical Center, New York, NY 10021, USA. 